\title{
Effect of Reduced Audibility on Mini-Mental State Examination Scores
}

DOI: $10.3766 /$ jaaa.17139

\author{
Laura Gaeta* \\ Jo Azzarello $\dagger$ \\ Jonathan Baldwin \\ Carrie A. Ciro§ \\ Mary A. Hudson 9 \\ Carole E. Johnsonq \\ Andrew B. John
}

\begin{abstract}
Background: The interaction of audition and cognition has been of interest to researchers and clinicians, especially as the prevalence of hearing loss and cognitive decline increases with advancing age. Cognitive screening tests are commonly used to assess cognitive status in individuals reporting changes in memory or function or to monitor cognitive status over time. These assessments are administered verbally, so performance may be adversely affected by hearing loss. Previous research on the impact of reduced audibility on cognitive screening test scores has been limited to older adults with sensorineural hearing loss (SNHL) or young adults with normal hearing and simulated audibility loss. No comparisons have been conducted to determine whether age-related SNHL and its impact on cognitive screening tests is successfully modeled by audibility reduction.
\end{abstract}

Purpose: The purpose of this study was to examine the effects of reduced audibility on the Mini-Mental State Examination (MMSE), a common bedside cognitive screening instrument, by comparing performance of cognitively normal older adults with SNHL and young adults with normal hearing.

Design: A 1:1 gender-matched case-control design was used for this study.

Study Sample: Thirty older adults (60-80 years old) with mild to moderately severe SNHL (cases) and 30 young adults (18-35 years old) with normal hearing (controls) served as participants for this study. Participants in both groups were selected for inclusion if their cognitive status was within normal limits on the Montreal Cognitive Assessment.

Data Collection and Analysis: Case participants were administered a recorded version of the MMSE in background noise at a signal-to-noise ratio of $+25-\mathrm{dB}$ SNR. Control participants were administered a digitally filtered version of the MMSE that reflected the loss of audibility (i.e., threshold elevation) of the matched case participant at a signal-to-noise ratio of $+25-\mathrm{dB}$ SNR. Performance on the MMSE was scored using standard criteria.

Results: Between-group analyses revealed no significant difference in the MMSE score. However, within-group analyses showed that education was a significant effect modifier for the case participants.

Conclusions: Reduced audibility has a negative effect on MMSE score in cognitively intact participants, which contributes to and confirms the findings of earlier studies. The findings suggest that observed reductions in score on the MMSE were primarily due to loss of audibility of the test item. The negative effects of audibility loss may be greater in individuals who have lower levels of educational attainment.

*Department of Communication Sciences and Disorders, College of Health and Human Services, California State University, Sacramento, CA; † College of Nursing, University of Oklahoma Health Sciences Center, Oklahoma City, OK; $\$$ Department of Medical Imaging and Radiation Sciences, College of Allied Health, University of Oklahoma Health Sciences Center, Oklahoma City, OK; §Department of Occupational Therapy Education, School of Health Professions, University of Kansas Medical Center, Kansas City, KS; $q$ Department of Communication Sciences and Disorders, College of Allied Health, University of Oklahoma Health Sciences Center, Oklahoma City, OK

Corresponding author: Laura Gaeta, Department of Communication Sciences and Disorders, College of Health and Human Services, California State University, Sacramento, CA 95819; Email: I.gaeta@csus.edu 
Higher levels of educational attainment may offset decreased performance on the MMSE because of reduced audibility. Failure to consider audibility and optimize communication when administering these assessments can lead to invalid results (e.g., false positives or missed information), misdiagnosis, and inappropriate recommendations for medication or intervention.

Key Words: aging, assessment, cognition, hearing loss

Abbreviations: $\mathrm{CDT}=$ clock drawing test; $\mathrm{Cl}=$ confidence interval; $\mathrm{RMS}=$ root-mean-square; MMSEMini-Mental State Examination; MoCA = Montreal Cognitive Assessment; SD = standard deviation; SNHL = sensorineural hearing loss; SNR = signal-to-noise ratio

A ging is associated with changes in hearing sensitivity and cognitive functioning, declining with advancing age. The prevalence of agerelated hearing loss and cognitive impairment is expected to rise in the general population, especially for those $>60$ years, in part because adults are living longer. In the United States, about $75 \%$ of adults aged $\geq 70$ years have hearing loss ranging in severity from mild to profound (Goman and Lin, 2016), making age-related hearing loss the most common sensory impairment in older adults. Age-related hearing loss, or presbycusis, is characterized as a progressive, bilateral, symmetrical sensorineural hearing loss (SNHL) that first affects sensitivity to high-frequency sounds (i.e., consonants) (Gelfand et al, 1986; Helfer and Wilber, 1990; PichoraFuller and Souza, 2003). Because of reduced hearing sensitivity within the high-frequency region, speech recognition is affected. Declines in speech understanding in older adults have been attributed to changes in the peripheral and central auditory system, as well as to various cognitive processes (Pichora-Fuller and Singh, 2006).

Cognitive changes with age can be classified as normal or pathological. Some cognitive domains, such as crystallized intelligence and vocabulary, improve with age whereas other domains, such as speed of processing and memory, decline (Harada et al, 2013). Declines in memory and other cognitive functions (e.g., calculations, judgment, and language) are features of dementia, a gradually progressive syndrome, which can limit a person's functional independence (Deary et al, 2009). Causes of dementia are often multifactorial, stemming from strokes (e.g., vascular dementia) or neurodegeneration (e.g., Alzheimer's disease), or may be associated with other disorders such as Parkinson's (e.g., Parkinson's disease dementia).

These age-related structural and physiological changes throughout the auditory and cognitive systems may interact in older adults, suggesting an association between hearing loss and cognitive decline. This association has been supported by longitudinal studies (Lin et al, 2011; Lin et al, 2013; Tomioka et al, 2015). Although the mechanism of this association is not yet fully established, hypotheses have been proposed that suggest that cognitive decline reduces the availability of resources available for understanding speech
(Schneider, 2011). In addition, those with age-related hearing loss are at a greater risk for developing dementia, indicating that age-related hearing loss is associated with incident dementia (Lin et al, 2011).

The association between age-related hearing loss and cognition has been studied through the use of speech recognition in noise testing. In adverse listening conditions, speech recognition is negatively affected and requires more effortful listening, leaving fewer cognitive resources for other functions such as working memory (Pichora-Fuller, 2003; Helfer and Freyman, 2008; Cousins et al, 2014; Carroll et al, 2016). Continuous effortful listening, such as in noisy environments, has been hypothesized to contribute to accelerated cognitive decline in older adults (Cardin, 2016).

In light of this recent evidence, the association between age-related hearing loss and cognitive decline is significant for clinicians evaluating older adults. Age-related hearing loss may have a negative impact on cognitive performance, thereby making diagnosis of cognitive impairment a challenge. Furthermore, the average adult waits $\geq 10$ years to be fit with hearing aids (Davis et al, 2007). This delay in seeking amplification can lead to sensory deprivation and cognitive decline, both of which contribute to social isolation (Monzani et al, 2008). Early detection of pathological changes associated with cognition is worsened by the lack of a definitive diagnostic tool and the complex multifactorial etiology. The estimated time-to-diagnosis for dementia is between 1 and 3 years (Cattel et al, 2000; Fiske et al, 2005). To diagnose pathological cognitive changes, providers usually rely on a brief cognitive screening test and caregiver report. These cognitive screenings are brief, psychometrically robust assessments that identify patients who require additional comprehensive cognitive assessments by a specialist, determine a differential diagnosis, or monitor progression of cognitive status (Woodford and George, 2007).

The Mini-Mental State Examination (MMSE; Folstein et al, 1975) is the most commonly used assessment of cognition by physicians (O'Bryant et al, 2008) and has been in use since 1975. The MMSE assesses a variety of cognitive domains, including but not limited to orientation, recall, language, and visual construction tasks (Folstein et al, 1975). It is psychometrically robust, having high test-retest reliability and sensitivity 
to moderate to severe stages of dementia (Tombaugh and McIntyre, 1992). Limitations of the MMSE include biases toward lower levels of education and reduced sensitivity for early or mild stages of dementia, resulting in ceiling effects and the possibility of diagnostic false negatives (Naugle and Kawczak, 1989; Simard, 1998).

The Montreal Cognitive Assessment (MoCA; Nasreddine et al, 2005) is a cognitive screening test that was developed to detect mild cognitive impairment to address the sensitivity limitation of the MMSE for earlier stages of dementia. The MoCA assesses multiple cognitive domains, such as executive function, sustained attention, working memory, and language (Nasreddine et al, 2005). Diagnosis of cognitive impairment with the MoCA has also been studied in various populations, including patients with stroke (Chiti and Pantoni, 2014) and Parkinson's disease (DalrympleAlford et al, 2010).

The MMSE, like most cognitive screening tests, is administered verbally, requiring the patient to respond appropriately to auditory information. Consequently, hearing loss can compromise the understanding of the verbally administered test items, instructions, or other information, and have a negative impact on the score on the cognitive screening test. Providers who are unaware of the patient's hearing loss or the impact of hearing loss on the verbally administered test items may not recognize errors due to reduced audibility and distinguish them from errors due to cognitive declines. This potential negative impact of hearing loss on the assessment of cognitive function emphasizes the need for timely diagnosis of hearing loss and cognitive status, in addition to the determination of hearing status before administering any assessment. Failure to confirm hearing status may lead to invalid results and diagnostic errors.

The impact of hearing loss on cognitive assessments has been explored in a limited number of studies. Dupuis et al (2015) studied the impact of dual sensory impairment (vision and hearing) on cognitive screening test scores. In the study, 301 older adults with hearing and vision impairments (50\% normal hearing and vision; 38\% hearing impairment; $5 \%$ vision impairment; $7 \%$ dual-sensory impairment) were administered the MoCA. Dupuis et al found that the participants with vision or hearing impairment performed worse on the MoCA than the participants with normal hearing and normal vision, providing evidence that sensory impairments can negatively impact performance on the MoCA. The findings of this study have implications for diagnosing cognitive decline as cognitive status may be underestimated in patients with sensory impairments.

Recently, Jorgensen et al (2016) investigated the effects of simulated audibility loss on MMSE scores. In the study, 125 young adults with normal hearing were randomized into five groups (normal hearing, mild to moderately severe sloping hearing loss, mild to severe sloping hearing loss, moderate to severe sloping hearing loss, and severe to profound sloping hearing loss) based on population data from Cruickshanks et al (1998). Loss of audibility for each group was simulated using Adobe Audition by filtering a recording of the MMSE that reflected each group's loss of audibility (i.e., threshold). Each participant was administered the digitally filtered MMSE, which was presented at 70-dB SPL in the presence of $45-\mathrm{dB}$ root-mean-square (RMS) spectrally shaped white noise. Jorgensen et al found that MMSE scores declined significantly as audibility was reduced among the participants. Jorgensen et al concluded that reduced audibility could lead to artificially low scores on the MMSE, even in young adults with normal hearing and cognition. However, despite the significant findings reported by Jorgensen et al (2016), the generalizability and clinical applications of the study are limited. Reduced audibility through digital filtering does not account for the distortion that accompanies SNHL in older adults, so it is unknown how the other components of SNHL affect comprehension of the cognitive screening test items.

The findings of Jorgensen et al, in addition to previous research, are not surprising given that patients must hear the test item correctly and respond to a verbally administered test question. Research on speech recognition has shown that noise can exacerbate the impact of hearing loss on performance on cognitive screening tests. The administration of a cognitive assessment in a noisy environment may lead to underestimated scores and, possibly, delayed identification of cognitive impairment and the risk of misdiagnosis.

The purpose of this study was to examine the impact of the distortion component of SNHL on cognitive screening test scores by simulating loss of audibility and comparing performance with a case-control design.

Based on the conclusions and limitations of Jorgensen et al's (2016) study, this study was needed to explore the possible influence of aging on a cognitive screening test by comparing performance of young adults with normal hearing and older adults with SNHL. Without this age-based comparison, previous studies were not able to conclude definitively that hearing loss beyond reduced audibility and age-related changes in cognition contributed to reduction in MMSE scores. In addition, comparing the performance of older and young adults allowed for further investigation into the distortional aspects of SNHL and its effects on speech understanding in older adults.

\section{METHODS}

$\Upsilon$ his study used a case-control design. Cases were defined as older adults (60-80 years old) with mild 
to moderately severe SNHL who use hearing aids, and the controls were defined as gender-matched young adults (18-35 years old) with normal hearing. All data were collected prospectively from January 24,2017 , to April 10, 2017. All study procedures were completed at the University of Oklahoma Health Sciences Center in a single-walled sound-treated booth that met American National Standards Institute S3.1 maximum permissible ambient noise levels (ANSI, 2007). A 3M SoundPro SE/DL Series Type II sound level meter (3M, St Paul, MN) positioned at the ear level when seated was used to establish presentation levels for the signal and noise channels on the recording. Calibration tones matched to the long-term RMS of the stimulus (MMSE) and noise (multitalker babble) signals were used at 70-dB SPL RMS amplitude. Digital audio files of MMSE recordings were routed from a desktop computer using Windows Media Player to a Madsen Orbiter 922 clinical audiometer to JBL Control 5 BP2X speakers (JBL by Harman, Stamford, $\mathrm{CT}$ ) in the booth via a two-channel Crown D75A amplifier (Crown Audio by Harman, Northridge, CA). The participant was seated $1 \mathrm{~m}$ from the signal speaker at $0^{\circ}$ azimuth (at head height), which simulated the ideal position of a clinician administering the MMSE face-to-face. Two additional JBL 5 BP2X bipolar speakers for the noise channel, positioned at $45^{\circ}$ and $135^{\circ}$ azimuth to the seated participant, were located at a distance of $1 \mathrm{~m}$ at head height. Distance and location between the participant and three speakers were visually confirmed for every participant using positional markers in the test space. Presentation levels were confirmed periodically throughout the data collection processes for consistency. A clipboard with the forms used for the MMSE test items that required responses on paper was positioned to the participant's nondominant hand side on a small stool.

Two groups of participants were recruited to serve as cases and controls. Control participants were recruited to match the gender of the case participants on a 1:1 basis. Thirty case participants (mean $=69.4$ years old, standard deviation $[\mathrm{SD}]=4.8$ years, range $=$ 61-78 years old) were recruited through local and oncampus clinics in Oklahoma City. Thirty control participants (mean $=24.2$ years old, $\mathrm{SD}=2.9$ years, range $=$ 20-32 years old) were recruited from the Oklahoma City metropolitan area and campus. All case participants were users of binaural hearing aids but participated in study assessments unaided to allow comparisons with the control participants. Case participants completed all pre-experimental tasks with the use of their hearing aids. Older and young adults were ineligible to participate if they had been diagnosed with a cognitive or neurological disorder (e.g., dementia, traumatic brain injury, and Parkinson's disease) by a physician, had been diagnosed with a learning disability, were unable to communicate verbally, did not have corrected vision, or were unable to complete all required tasks.

To isolate the impact of audibility loss on cognitive screening test scores, participants were administered the MoCA and had to score $\geq 26$ on the MoCA to participate; no participants scored $<26$. The MoCA was used for determining inclusion eligibility because of its higher sensitivity than the MMSE for distinguishing mild cognitive impairment (Nasreddine et al, 2005; Roalf et al, 2013) and for comparison with Jorgensen et al (2016), which used the MMSE as the main study measure.

This study was approved by the Institutional Review Board at the University of Oklahoma Health Sciences Center in December 2016.

\section{Methodology}

All participants completed a case history form with the investigator before beginning study measures. All participants underwent otoscopy, tympanometry, puretone audiometry, and the QuickSIN (Etymotic Research, Elkgrove Village, IL). The average audiogram for case participants revealed a mild sloping to moderately severe SNHL, and the average audiogram for control participants showed normal hearing sensitivity (see Figure 1).

Adapted from methodology used by Jorgensen et al (2016), participants in both groups were asked to rate their familiarity with the following cognitive assessments to ensure that performance was unaffected by memory of the test items: MoCA, Abbreviated Mental Test Score, MMSE, St. Louis University Mental Status Examination, and the clock drawing test (CDT). Two of the assessments, the MMSE and the MoCA, were used as study measures. To establish familiarity with the MMSE and MoCA, the other assessments (Abbreviated Mental Test Score, St. Louis University Mental Status Examination, and CDT) were included as foils. The CDT was the most familiar test to the participants in both groups; however, no participants reported high levels of familiarity with the other tests, including the MMSE, confirming low familiarity with the study measure.

\section{Stimuli}

A male volunteer with a neutral accent recorded the test questions from the MMSE as the original stimuli, which was used as the stimulus for the case participants. Test items were equalized to $60 \mathrm{~dB}(\mathrm{~A})$ based on the average amplitude RMS of the original recordings analyzed in Adobe Audition CS6, v. 5.0. Multitalker babble (Auditec of St. Louis, 1971) was presented at $35 \mathrm{~dB}(\mathrm{~A})$ and added to the second channel to simulate typical background noise levels during 


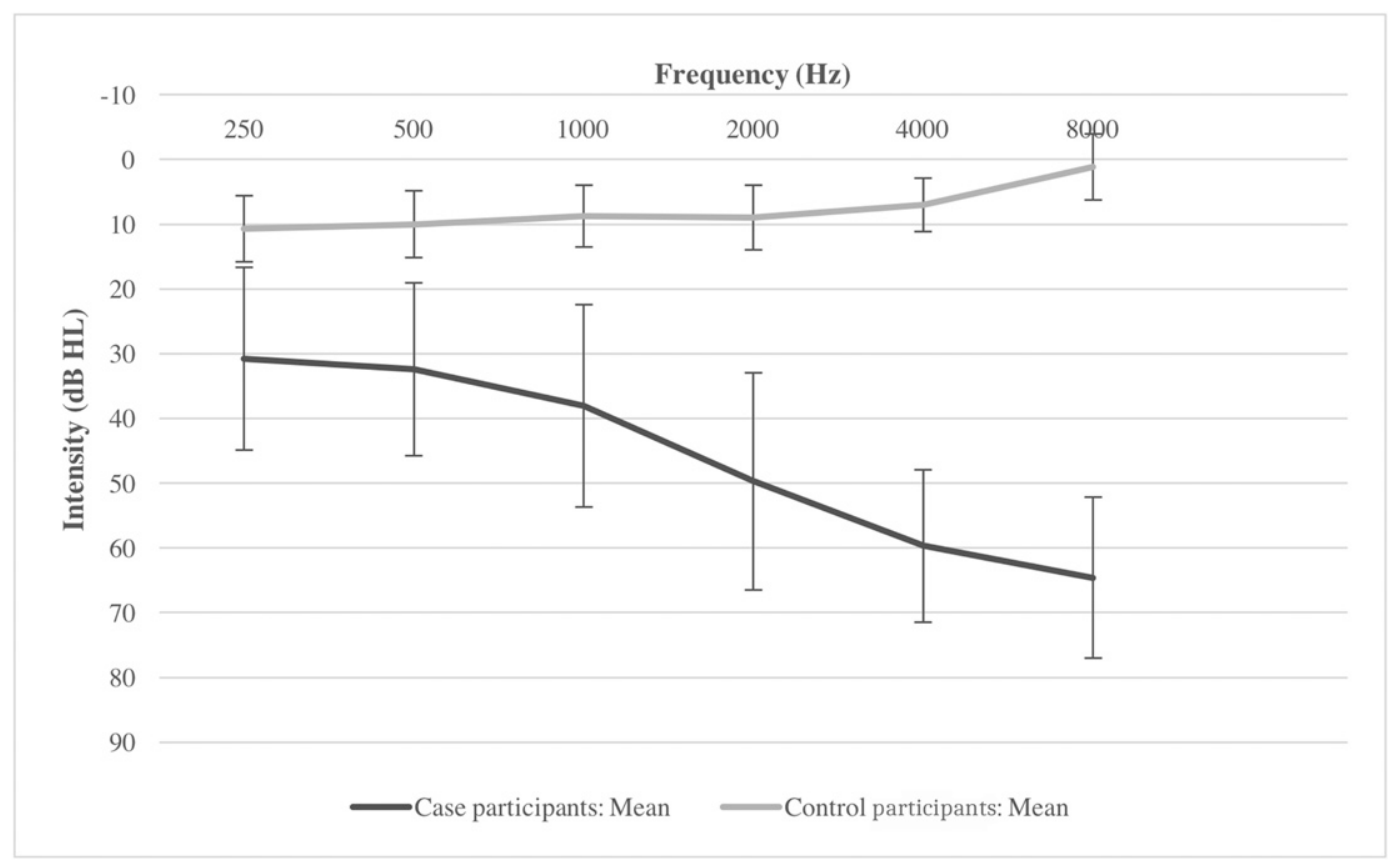

Figure 1. Average audiometric thresholds for case and control participants (error bars represent one SD) before simulation of audibility loss. After simulation of audibility loss, thresholds for case and control participants were identical.

administration of the MMSE in a clinical setting and for comparison with Jorgensen et al (2016). Multitalker babble is more ecologically valid than white noise for simulating clinical settings because of the informational and energetic masking components (Killion et al, 2004). Both groups of participants were administered the MMSE in the presence of background noise (+25-dB SNR).

The original MMSE recordings were used for the case participants. The case participants were administered the MMSE without the use of their hearing aids. For the control participants, MMSE recordings were digitally filtered to simulate a matched loss of audibility. Loss of audibility was simulated by attenuating the stimuli recording in various octave bands by the difference in the unaided thresholds between the case participant and the matched control participant using Adobe Audition CS6, v. 5.0. Simulated loss of audibility for the control participants was calculated as the difference in better-ear unaided thresholds at 250, 500, 1000, 2000, $3000,4000,6000$, and $8000 \mathrm{~Hz}$ for the control participants and the matched case participants. This method controlled for audibility loss between the two groups. This recording was used with the control participants in the simulated unaided condition.

The MMSE test items were preceded and followed by a set of five unscored but similarly phrased general knowledge questions. These ten questions allowed the participant to become familiar with the task and allowed the tester to confirm equipment function.

Test items were presented in the same sequence as the original MMSE and were not repeated. Before pre- senting the recorded test questions, instructions were provided to each participant. The recording of the MMSE questions was paused after each test item to allow the participant to respond before presenting the next question. No human tester was in the booth with the participant, so all test items that required a visual cue were presented via PowerPoint slideshow on a computer screen in the sound booth.

\section{Test Levels}

A signal-to-noise ratio (SNR) in the sound field test environment was set to +25-dB SNR, which was consistent with Jorgensen et al (2016), and to simulate a favorable listening environment. The test items (signal) were presented at $60 \mathrm{~dB}(\mathrm{~A})$, representative of conversational level speech, and the multitalker babble (noise) was presented at $35 \mathrm{~dB}(\mathrm{~A})$, or typical noise levels in a quiet room.

\section{Test Scoring}

Audio recordings of all sessions were used to confirm responses after the study appointment. Responses for all participants were scored based on their first or self-corrected response according to the instructions provided on the original MMSE as a score out of 30 points. Participants were not informed of their errors during testing and mistakes were not corrected. Scores were categorized and interpreted based on the cutoff values provided by Mungas (1991) (see Table 1). Scores were not adjusted for education level because all of the 
Table 1. MMSE Score Interpretation (Mungas, 1991)

\begin{tabular}{ll}
\hline MMSE Score Ranges & \multicolumn{1}{c}{ Interpretation } \\
\hline $25-30$ & Normal \\
$21-24$ & Mild impairment \\
$10-20$ & Moderate impairment \\
$0-9$ & Severe impairment \\
\hline
\end{tabular}

participants had at least a high school education. Participants were unaware of the goal of the testing until the end of the study appointment.

\section{RESULTS}

A ll data were analyzed using SAS 9.4 (Cary, NC). An alpha of 0.05 was established a priori for the statistical analyses. Analyses were conducted for withinand between-group differences.

\section{QuickSIN}

The QuickSIN was administered to all participants to quantify SNR loss. QuickSIN scores were calculated following the scoring procedures provided by Etymotic Research (2001). Mean SNR loss was 7.95-dB SNR $(\mathrm{SD}=5.79$; median $=6.25$; minimum $=0.5 ;$ maximum $=$ 20) for case participants and 0.68-dB SNR (SD = 1.15; median $=1.0$; minimum $=21.5$; maximum $=3.0$ ) for control participants. As expected, QuickSIN scores for the case participants were higher (i.e., required a greater SNR) than those for the control participants. QuickSIN score was a significant covariate and was adjusted for in the analysis $(F=16.68, p=0.0003)$.

\section{Effect of Reduced Audibility on MMSE Score}

A linear mixed model, which allowed for assessment of possible confounding variables and/or effect modifiers and accounted for correlation among the matched case and control participants, was used to analyzed differences in MMSE scores among both groups of participants. Mean MMSE scores for the case participants and control participants are shown in Table 2 and Figure 2.

There were no statistically significant differences in MMSE scores between the case and control participants, indicating that audibility is the primary explanation for the observed decrease in MMSE scores. Education level was the only significant effect modifier in the relationship between the case and control participants and MMSE score $(F=9.05, p=0.0070)$, so MMSE scores among case and control participants were stratified by education level (less than a bachelor's degree; a bachelor's degree or higher). Among individuals with less than a bachelor's degree, case participants scored 12.0 points $[t=-4.30,95 \%$ confidence interval (CI): $6.0,18.0, p=0.0007]$ lower on the MMSE compared with control participants. Among participants with a bachelor's degree or higher, there was no significant difference in MMSE score for both groups $(t=$ $0.91, p=0.3709$ ). Figure 3 shows mean MMSE scores for each group by education level.

Figure 4 illustrates the relationship between the MoCA score and the MMSE score among case and control participants. Performance on the MoCA was not associated with the MMSE score for the case participants ( $\beta=1.84, \beta 95 \% \mathrm{CI}:-1.19,4.88, t=1.24, p=0.2247)$ or the control participants $(\beta=-0.87, \beta 95 \% \mathrm{CI}:-3.71$, $1.96, t=-0.63, p=0.5328$ ). Across groups, MoCA performance was not associated with MMSE score $(\beta=0.74, \beta 95 \% \mathrm{CI}:-1.03,2.52, t=0.84, p=$ 0.4062 ).

High-frequency pure-tone average was calculated based on the better-ear thresholds at 1000, 2000, and $4000 \mathrm{~Hz}$. MMSE scores decreased one point (95\% CI: $0.06,1.9)$ for every $10-\mathrm{dB}$ increase in the high-frequency pure-tone average $(\beta=-0.10, t=2.08, p=0.0416)$.

MMSE scores were interpreted (based on Mungas, 1991) for both groups using the severity categories provided in Table 1 as normal, mild, moderate, or severe impairment. Even though all participants had MoCA scores that were within normal limits at the time of enrollment, participants in both groups had scores on the MMSE that were classified as either mild, moderate, or severe cognitive impairment (see Figure 5), suggesting that audibility loss alone may result in erroneous classification of cognitive status.

\section{DISCUSSION}

lthough it was hypothesized that age and distorA tional factors would result in worse performance among case participants than control participants, there were no significant differences between the two groups of participants. The present study was based on the work by Jorgensen et al (2016), which found an association between reduced audibility and poor MMSE scores in young adults with normal hearing.

Table 2. Descriptive Statistics for MMSE Scores Obtained by Study Participants

\begin{tabular}{lcccccc}
\hline & $N$ & Mean & SD & Median & Minimum & Maximum \\
\hline Case participants & 30 & 21.9 & 8.3 & 25.5 & 1.0 & 30.0 \\
Control participants & 30 & 24.3 & 8.2 & 28.0 & 1.0 & 30.0 \\
\hline
\end{tabular}




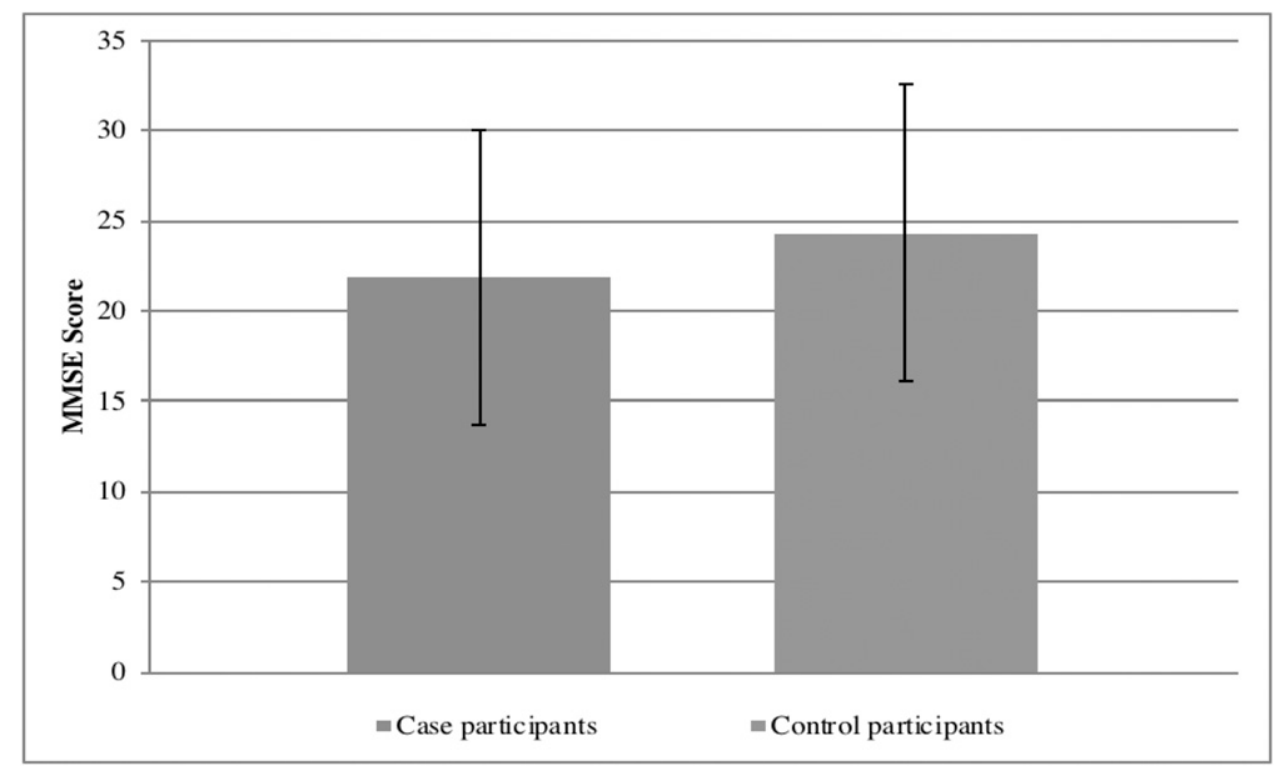

Figure 2. Comparison of mean MMSE scores by group (error bars represent one SD).

Jorgensen et al (2016) selected young adults to control for aging effects on the auditory system but noted that older adults with hearing loss may not have similar performance as the young adults because of these aging effects. The present study addressed this need through comparisons of performance on the MMSE for young adults with normal hearing and older adults with SNHL. Based on the findings, decreases in performance on the MMSE were primarily the result of reduced audibility and its effect on understanding of the test items. Based on the QuickSIN scores of each group, any group differences, if present, would not be adequately ex- plained by SNR loss. This absence may be attributed to the +25-dB SNR used in this study, which did not sufficiently assess the participants' ability to distinguish speech in noise.

Cognitive screening tests, ideally, should be free from biases related to age, gender, socioeconomic status, culture, and education, but previous research has shown that poorer scores on cognitive screening tests are associated with older age and lower levels of education (Ganguli et al, 2010). This bias can ultimately impact the interpretation of test results in the elderly or in populations that have lower levels of educational

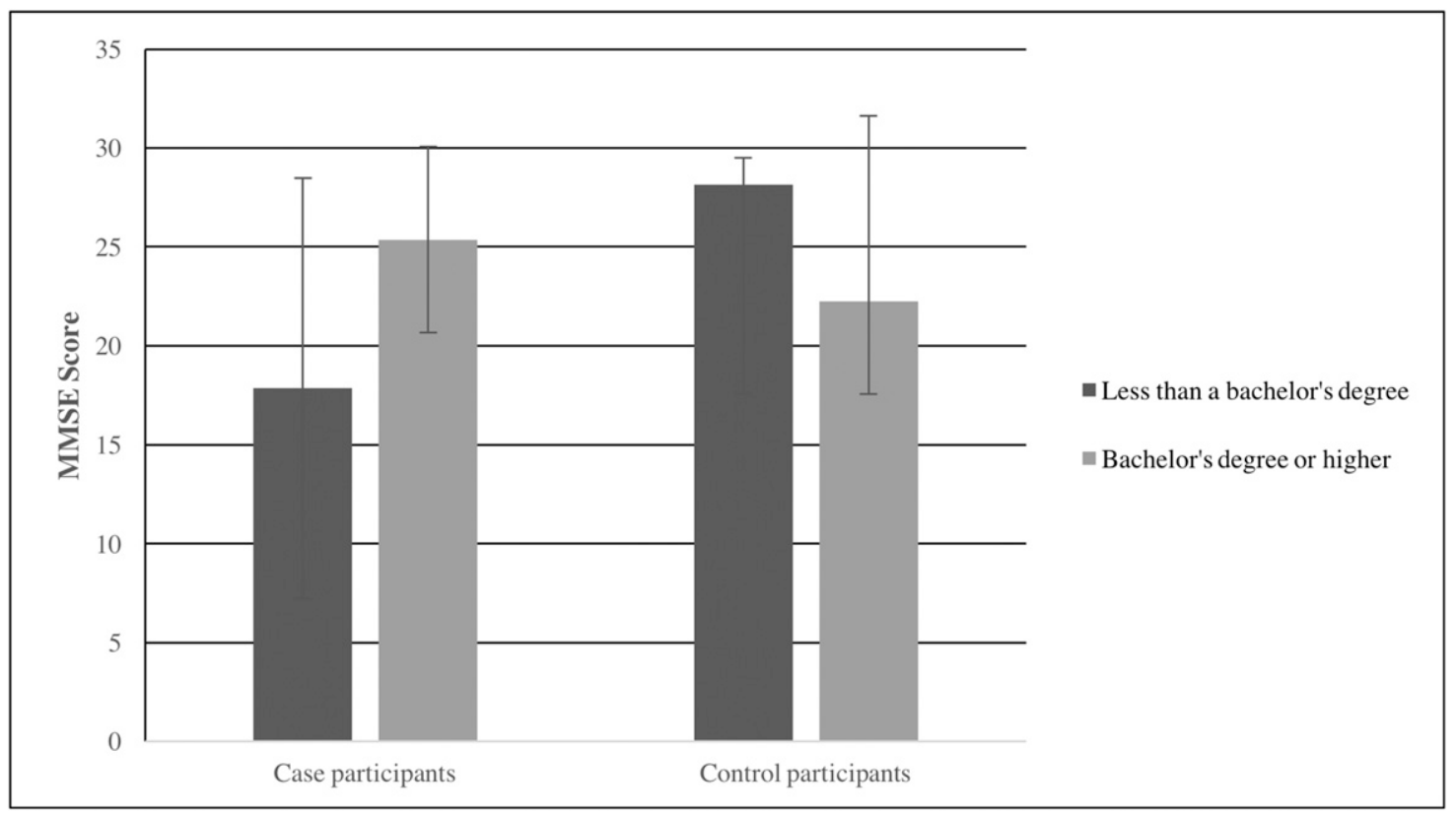

Figure 3. Comparison of mean MMSE scores by group and education level (error bars represent one SD). 


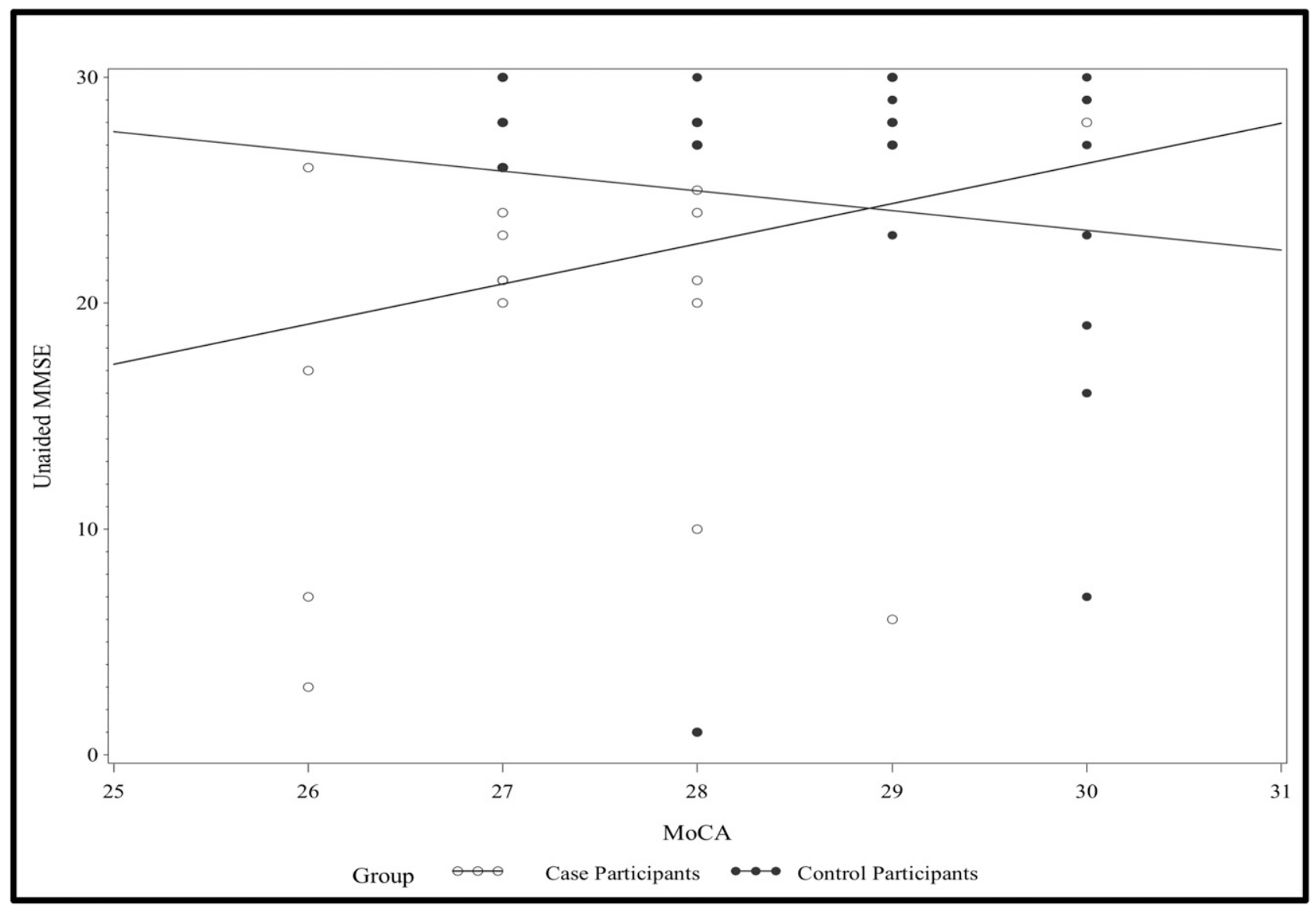

Figure 4. Relationship between performance on the MoCA and MMSE by group.

attainment. For example, providers may find interpretation a challenge if that patient is older with less than a high school education, even before considering the potential impact of a patient's hearing loss.

Participants who had higher levels of educational attainment had greater scores on the MMSE than participants with lower levels of education. This difference in MMSE score by education level may be explained by the highly educated participants' use of context when listening to the MMSE test items. Those with lower levels of educational attainment may not have efficiently used context cues from the MMSE test items, which could contribute to difficulties in speech understanding beyond those associated with loss of audibility. Therefore, higher levels of education may be able to partially compensate for any information degraded by SNHL.

Despite all participants in the study scoring within normal limits on the MoCA, more than one-third of participants in the study had scores that would be interpreted as below normal on the MMSE. Fourteen case participants had MMSE scores that were categorized as mild, moderate, or severe impairment. For the control participants, eight participants had scores below normal. In both groups, individuals with actual and simulated hearing loss appeared to have a cognitive im- pairment. This finding underscores the need to confirm audibility before administering cognitive screening tests as those with cognition within normal limits may have artificially low scores on the MMSE solely because of reduced audibility. Audibility should always be confirmed or amplification provided before administering a cognitive screening test. If the cutoff values for highly educated adults were used (as recommended by O'Bryant et al [2008]), an additional six participants (five case participants and one control participant) would have scores that would be categorized as below normal. In this instance, highly educated adults would be incorrectly classified and diagnosed with at least a mild cognitive impairment solely because of reduced audibility of test questions. In the elderly and those with either low or very high levels of education, accurate interpretation and timely diagnosis is of even greater significance.

Biases toward lower levels of education are known limitations of the MMSE. Within-group analyses revealed that education was a significant effect modifier for the case participants, but this effect was not observed for the control participants. This difference suggests that biases toward lower levels of education may be more apparent if older adults are not using 


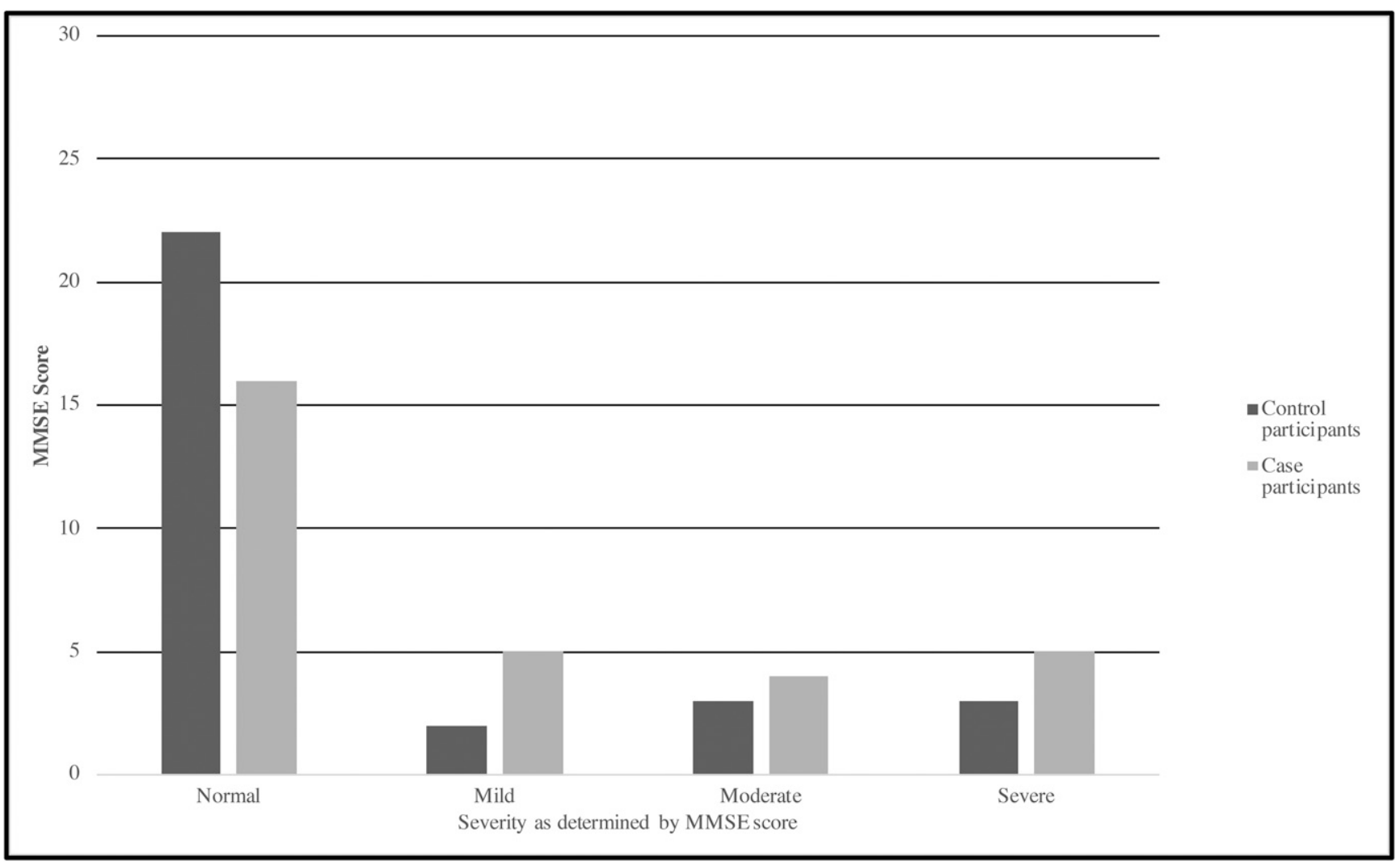

Figure 5. MMSE score interpretation by group.

amplification. It is reasonable to expect that patients with lower levels of education are also less likely to have access to hearing health care, which increases the likelihood that these patients may be overdiagnosed with cognitive impairment (Barnett et al, 2017; Chan et al, 2017).

Health-care providers who administer cognitive screening tests may be unaware of the interaction of hearing loss and cognitive decline or be trained to recognize signs of hearing loss, which can lead to misdiagnosis and/or artificially low scores on cognitive screening tests. Within the medical community, hearing loss has not been commonly considered a factor when communicating with older patients. Cohen et al's (2017) review found that only 16 of the 67 articles stated hearing loss as an important consideration for patient-doctor communication. These findings are surprising and unexpected because of the emphasis on effective communication and its role in the physician-patient relationship. Hearing loss, a barrier to successful and effective communication, is highly prevalent in older adults but was not a major consideration for providers. Audiologists can collaborate with physicians to advocate for early identification and appropriate management of hearing loss to increase awareness of the signs of sensory impairment in the medical community. Providers who are aware of the impact of hearing loss on communication may make appropriate referrals to audiologists and suggest ways to improve communication with patients, which ultimately contributes to better quality-of-care measures and communication. Diagnostic errors can be avoided through effective communication between the patient and provider.

Early diagnosis of cognitive disorders, such as dementia, is imperative for beginning intervention, recommending treatment, and reducing patient and caregiver burden over time. Interpretation of scores on cognitive screening tests is used as the basis for establishing baseline function and initiating intervention, in addition to patient and caregiver report. The provider must interpret and categorize the score as normal or abnormal, and, if abnormal, determine the severity of the impairment. The present study demonstrated that some individuals had scores that were considered below normal limits solely on the basis of reduced audibility. This finding indicates that, if not considered by the provider, a patient could be misdiagnosed with a cognitive impairment based on the erroneous interpretation of the test score and may then be referred unnecessarily, undergo additional testing, and inappropriate recommendations for interventions and/or medication. This study, along with Jorgensen et al (2016), demonstrated that 
performance on the MMSE can be adversely affected by the loss of audibility. Therefore, the diagnostic accuracy of the MMSE should be examined for administration to older patients with hearing loss. Misdiagnoses of dementia have been shown to cause increased expenses and harm to the patient, as well as greater caregiver burden (Graber et al, 2005; Gandhi et al, 2006; Singh et al, 2006).

Misdiagnosis and medical errors may also be due to poor provider-patient communication (Lingard et al, 2004). In a survey of patients who were deaf or hard of hearing, participants reported concerns regarding medications and procedures because of inadequate communication (Iezzoni et al, 2004). It was recommended that providers ask patients about their preferred mode to avoid miscommunication (Iezzoni et al, 2003). Patients who talk with their physician about their hearing are more likely to have an audiologic evolution (Nash et al, 2013), highlighting the importance of the primary care physician's role in the initial access to hearing health care.

Lastly, as most cognitive screening tests are administered verbally, hearing loss may compromise the comprehension of the test items, impacting performance and score on a cognitive screening test. Earlier research, including the present study, has provided evidence of the negative impact of audibility on cognitive screening scores. To address concerns of this impact of hearing loss on cognitive screening test scores, Uhlmann et al (1989) suggested the use of a written version of the MMSE. The written version of the MMSE was administered to patients with dementia with and without hearing loss. However, the patients with dementia and hearing loss had poorer scores on the written version of the MMSE compared with patients with normal hearing, further emphasizing the negative effect of hearing loss on cognitive screening test administration and scores.

\section{CONCLUSION}

$\mathrm{T}$ he present study examined the distortion associated with aging in the auditory system on cognitive screening test (MMSE) scores in older adults with hearing loss and young adults with normal hearing. Although there were no between-group differences, MMSE scores were negatively impacted by reduced audibility, leading to erroneous interpretation of test scores in cognitively intact individuals. Biases toward lower levels of education were also demonstrated, highlighting the need to further advocate and include amplification for older adults who have lower levels of educational attainment. Providers should be aware of the signs of age-related hearing loss and be able to distinguish these signs from those of cognitive decline. Audibility should always be optimized through the use of hearing aids and/or a personal listening device and confirmed to improve communication so that sensory changes, such as hearing loss, do not negatively impact performance on cognitive screening tests, thereby reducing the risk of delayed or incorrect diagnosis of a cognitive disorder.

\section{REFERENCES}

American National Standards Institute (ANSI). (2007) Methods for the Calculation of the Speech Intelligibility Index (ANSI S3.5-1997 [R2007]). New York, NY: Acoustical Society of America.

Auditec of St. Louis. (1971) "Multi-Talker Babble." [Audio CD]. St. Louis, MO: Auditec of St. Louis.

Barnett M, Hixon B, Okwiri N, Irungu C, Ayugi J, Thompson R, Bush ML. (2017) Factors involved in access and utilization of adult hearing healthcare: a systematic review. Laryngoscope 127:1187-1194.

Cardin V. (2016) Effects of aging and adult-onset hearing loss on cortical auditory regions. Front Neurosci 10:199.

Carroll R, Warzybok A, Kollmeier B, Ruigendijk E. (2016) Agerelated differences in lexical access relate to speech recognition in noise. Front Psychol 7:990.

Cattel C, Gambassi G, Sgadari A, Zuccalà G, Carbonin P, Bernabei R. (2000) Correlates of delayed referral for the diagnosis of dementia in an outpatient population. J Gerontol A Biol Sci Med Sci 55:M98-M102.

Chan S, Hixon B, Adkins M, Shinn JB, Bush ML. (2017) Rurality and determinants of hearing healthcare in adult hearing aid recipients. Laryngoscope 127:2362-2367.

Chiti G, Pantoni L. (2014) Use of Montreal Cognitive Assessment in patients with stroke. Stroke 45:3135-3140.

Cohen JM, Blustein J, Weinstein BE, Dischinger H, Sherman S, Grudzen C, Chodosh J. (2017) Studies of physician-patient communication with older patients: how often is hearing loss considered? A systematic literature review. J Am Geriatr Soc 65:1642-1649.

Cousins KA, Dar H, Wingfield A, Miller P. (2014) Acoustic masking disrupts time-dependent mechanisms of memory encoding in word-list recall. Mem Cognit 42:622-638.

Cruickshanks KJ, Wiley TL, Tweed TS, Klein BE, Klein R, MaresPerlman JA, Nondahl DM. (1998) Prevalence of hearing loss in older adults in Beaver Dam, Wisconsin. The epidemiology of hearing loss study. Am J Epidemiol 148:879-886.

Dalrymple-Alford JC, MacAskill MR, Nakas CT, Livingston L, Graham C, Crucian GP, Melzer TR, Kirwan J, Keenan R, Wells S, Porter RJ, Watts R, Anderson TJ. (2010) The MoCA: well-suited screen for cognitive impairment in Parkinson disease. Neurology 75:1717-1725.

Davis A, Smith P, Ferguson M, Stephens D, Gianopolulos I. (2007) Acceptability, benefit and costs of early screening for hearing disability: a study of potential screening tests and models. Health Technol Assess 11:1-294.

Deary IJ, Corley J, Gow AJ, Harris SE, Houlihan LM, Marioni RE, Penke L, Rafnsson SB, Starr JM. (2009) Age-associated cognitive decline. Br Med Bull 92:135-152. 
Dupuis K, Pichora-Fuller MK, Chasteen AL, Marchuk V, Singh G, Smith SL. (2015) Effects of hearing and vision impairments on the Montreal Cognitive Assessment. Neuropsychol Dev Cogn B Aging Neuropsychol Cogn 22:413-437.

Etymotic Research. (2001) Quick Speech-in-Noise Test [Audio $C D]$. Elk Grove Village, IL: Etymotic Research.

Fiske A, Gatz M, Aadnøy B, Pedersen NL. (2005) Assessing age of dementia onset: validity of informant reports. Alzheimer Dis Assoc Disord 19:128-134.

Folstein MF, Folstein SE, McHugh PR. (1975) "Mini-mental state." A practical method for grading the cognitive state of patients for the clinician. J Psychiatr Res 12:189-198.

Gandhi TK, Kachalia A, Thomas EJ, Puopolo AL, Yoon C, Brennan TA, Studdert DM. (2006) Missed and delayed diagnoses in the ambulatory setting: a study of closed malpractice claims. Ann Intern Med 145:488-498.

Ganguli M, Snitz BE, Lee CW, Vanderbilt J, Saxton JA, Chang CC. (2010) Age and education effects and norms on a cognitive test battery from a population-based cohort: the MonongahelaYoughiogheny Healthy Aging Team. Aging Ment Health 14:100-107.

Gelfand SA, Piper N, Silman S. (1986) Consonant recognition in quiet and in noise with aging among normal hearing listeners. J Acoust Soc Am 80:1589-1598.

Goman AM, Lin FR. (2016) Prevalence of hearing loss by severity in the United States. Am J Public Health 106:1820-1822.

Graber ML, Franklin N, Gordon R. (2005) Diagnostic error in internal medicine. Arch Intern Med 165:1493-1499.

Harada CN, Natelson Love MC, Triebel KL. (2013) Normal cognitive aging. Clin Geriatr Med 29:737-752.

Helfer KS, Freyman RL. (2008) Aging and speech-on-speech masking. Ear Hear 29:87-98.

Helfer KS, Wilber LA. (1990) Hearing loss, aging, and speech perception in reverberation and noise. J Speech Hear Res 33:149-155.

Iezzoni L, Davis RB, Soukup J, O'Day B. (2003) Quality dimensions that most concern people with physical and sensory disabilities. Arch Intern Med 163:2085-2092.

Iezzoni L, O’Day B, Killeen M, Harker H. (2004) Communicating about health care: observations from persons who are deaf or hard of hearing. Ann Intern Med 140:356-262.

Jorgensen LE, Palmer CV, Pratt S, Erickson KI, Moncrieff D. (2016) The effect of decreased audibility on MMSE performance: a measure commonly used for diagnosing dementia. J Am Acad Audiol 27:311-323.

Killion MC, Niquette PA, Gudmundsen GI, Revit LJ, Banerjee S. (2004) Development of quick speech-in-noise test for measuring signal-to-noise ratio loss in normal-hearing and hearing-impaired listeners. J Acoust Soc Am 116:2395-2405.

Lin FR, Ferrucci L, Metter EJ, An Y, Zonderman AB, Resnick SM. (2011) Hearing loss and cognition in the Baltimore Longitudinal Study of Aging. Neuropsychology 25:763-770.

Lin FR, Yaffe K, Xia J, Xue QL, Harris TB, Purchase-Helzner E, Satterfield S, Ayonayon HN, Ferrucci L, Simonsick EM; Health ABC Study Group. (2013) Hearing loss and cognitive decline in older adults. JAMA Intern Med 173:293-299.
Lingard L, Espin S, Whyte S, Regehr G, Baker GR, Reznick R, Bohnen J, Orser B, Doran D, Grober E. (2004) Communication failures in the operating room: an observational classification of recurrent types and effects. Qual Saf Health Care 13: $330-334$.

Monzani D, Galeazzi GM, Genovese E, Marrara A, Martini A. (2008) Psychological profile and social behaviour of working adults with mild to moderate hearing loss. Acta Otorhinolaryngol Ital 28: 61-66.

Mungas D. (1991) In-office mental status testing: a practical guide. Geriatrics 46:54-56.

Nash SD, Cruickshanks KJ, Huang GH, Klein BE, Klein R, Nieto FJ, Tweed TS. (2013) Unmet hearing health care needs: the beaver Dam offspring study. Am J Public Health 103:1134-1139.

Nasreddine ZS, Phillips NA, Bédirian V, Charbonneau S, Whitehead V, Collin I, Cummings JL, Chertkow H. (2005) The Montreal Cognitive Assessment, MoCA: a brief screening tool for mild cognitive impairment. J Am Geriatr Soc 53:695-699.

Naugle RI, Kawczak K. (1989) Limitations of the Mini-Mental State Examination. Cleve Clin J Med 56:277-281.

O'Bryant SE, Humphreys JD, Smith GE, Ivnik RJ, GraffRadford NR, Petersen RC, Lucas JA. (2008) Detecting dementia with the Mini-Mental State Examination in highly educated individuals. Arch Neurol 65:963-967.

Pichora-Fuller MK. (2003) Cognitive aging and auditory information processing. Int $J$ Audiol 42:2S26-2S32.

Pichora-Fuller MK, Singh G. (2006) Effects of age on auditory and cognitive processing: Implications for hearing aid fitting and audiologic rehabilitation. Trends Amplif 10(1):29-59.

Pichora-Fuller MK, Souza PE. (2003) Effects of aging on auditory processing of speech. Int $J$ Audiol 42:11-16.

Roalf DR, Moberg PJ, Xie SX, Wolk DA, Moelter ST, Arnold SE. (2013) Comparative accuracies of two common screening instruments for the classification of Alzheimer's disease, mild cognitive impairment and healthy aging. Alzheimers Dement 9:529-537.

Schneider BA. (2011) How age affects auditory-cognitive interactions in speech comprehension. Audiol Res 1:e10.

Simard M. (1998) The Mini-Mental State Examination: strengths and weaknesses of a clinical instrument. Can Rev Alzheimers Dis Other Demen 2:10-12.

Singh H, Petersen LA, Thomas EJ. (2006) Understanding diagnostic errors in medicine: a lesson from aviation. Qual Saf Health Care 15:159-164.

Tombaugh TN, McIntyre NJ. (1992) The mini-mental state examination: a comprehensive review. J Am Geriatr Soc 40:922-935.

Tomioka K, Okamoto N, Morikawa M, Kurumatani N. (2015) Self-reported hearing loss predicts 5-year decline in higher-level functional capacity in high-functioning elderly adults: the FujiwaraKyo study. J Am Geriatr Soc 63:2260-2268.

Uhlmann RF, Teri L, Rees TS, Mozlowski KJ, Larson EB. (1989) Impact of mild to moderate hearing loss on mental status testing. Comparability of standard and written mini-mental state examinations. J Am Geriatr Soc 37:223-228.

Woodford HJ, George J. (2007) Cognitive assessment in the elderly: a review of clinical methods. QJM 100:469-484. 